Pacific Journal of Mathematics

APPLICATIONS OF ULTRAPRODUCTS OF PAIRS OF

Co 


\title{
APPLICATIONS OF ULTRAPRODUCTS OF PAIRS OF CARDINALS TO THE THEORY OF MODELS
}

\author{
C. C. Chang and H. Jerome Keisler
}

Introduction. Recently in [8] Vaught introduced the interesting notion of a pair of cardinals $\left(\kappa(A), \kappa\left(R_{0}\right)\right)$ for a model $\left\langle A, R_{0}, \cdots\right\rangle$ of a given first-order theory with identity. He proved that if a theory (with countably many nonlogical constants) has a model with a pair of cardinals $(\alpha, \beta)$ where $\omega \leqq \beta<\alpha$, then it has a model with the pair of cardinals $\left(\omega_{1}, \omega_{0}\right)$. In this paper we have obtained a number of results along the same lines (they may be found in detail in $\S 4$ ); roughly speaking, our results are concerned with increasing one or both of the cardinals in the pair $(\alpha, \beta)$.

It turns out that most of our results on pairs of cardinals of a model are simple consequences of set-theoretical theorems concerning ultraproducts, ultrapowers, and limit ultrapowers of pairs of cardinals. We have isolated these set-theoretical theorems in $\S 2$ and $\S 3$, where they are presented with no reference to model theory.

In the last section of the paper, we give some counterexamples to certain plausible conjectures analogous to Vaught's and our results. We conclude the paper by stating a number of open problems. We wish to make it clear here that we do not claim to have originated all of these problems; in view of Vaught's result, some of them arise quite naturally and undoubtedly have been considered before.

1. Preliminaries. We employ the usual symbols $\varepsilon, \subseteq, \cap, \cup, \cap$, $U$, to denote the various familiar set-theoretical notions. The expression $\{t \mid \phi(t)\}$ shall denote the set of all elements $t$ such that $\phi(t)$ holds. Ordinal numbers will be denoted by $\xi, \zeta, \eta$, and natural numbers (finite ordinal numbers) by $m, n, p$. The symbols $0,1,2, \cdots$, denote the first natural numbers. We suppose the ordinals have been defined so that each ordinal coincides with the set of all smaller ordinals. Thus in particular 0 is the empty set. We identify cardinal numbers with the corresponding initial ordinal numbers. The letters $\alpha, \beta, \gamma, \delta$, denote arbitrary cardinals; $\omega$ denotes the smallest infinite cardinal; $\alpha^{+}$denotes the smallest cardinal greater than $\alpha$. For each ordinal $\xi, \omega_{\xi}$ denotes the smallest infinite cardinal which exceeds $\omega_{\zeta}$ for each $\zeta<\xi$. The cofinality of the cardinal $\alpha$ is denoted by $c f(\alpha)$. (See [6] for its definition

Received November 6, 1961. The results of this paper have been previously announced in [1]. The authors became interested in this problem through R. L. Vaught. The preparation of the paper was supported in the case of the first-named author by the National Science Foundation under grant G-14092. 
and elementary properties.) The notion of the sum $\xi+\zeta$ of two ordinals $\xi, \zeta$ is assumed to be known. Let $\alpha_{i}$ be a cardinal for each $i \in I ; \sum_{i \in I} \alpha_{i}$ and $\prod_{i \in I} \alpha_{i}$ denote, respectively, their cardinal sum and cardinal product. $\alpha^{\beta}$ shall denote the cardinal $\alpha$ to the power $\beta ; \alpha^{\beta}$ shall denote the cardinal $\alpha$ to the weak power $\beta$, i.e., $\sum_{\gamma<\beta} \alpha^{\gamma}$. Let $X, I$, and $X_{i}$ for each $i \in I$ be arbitrary sets. $\mathscr{\mathscr { P }}_{i \in I} X_{i}$ denotes the cartesian product of the sets $X_{i}$ with $i \in I$, and $\kappa(X)$ denotes the cardinal of $X$. We assume the reader is familiar with the notions of a filter on $I$, and an ultrafilter on $I$. Let $D$ be an ultrafilter on $I$. For any functions $f, g \in \mathscr{T}_{i \in I} \alpha_{i}$, we write $f \equiv_{D} g$ (read $f$ and $g$ are equivalent modulo $\left.D\right)$ if $\{i \in I \mid f(i)=$ $g(i)\} \in D$. The statement $f \equiv_{D} g$ has the intuitive meaning that $f$ and $g$ are equal almost everywhere. It is proved in [2] that $\equiv_{D}$ is an equivalence relation on $\mathscr{P}_{i \in I} \alpha_{i}$. For each $f \in \mathscr{P}_{i \in I} \alpha_{i}$, let $f \mid D=\left\{g \mid g \equiv_{D} f\right\}$, the equivalence class of $f$ with respect to $D$. By the (cardinal) ultraproduct of the cardinals $\alpha_{i}$ modulo $D$, in symbols, $\prod_{i \in I} \alpha_{i} / D$, we mean the cardinal $\kappa\left\{f|D| f \in \mathscr{F}_{i \in I} \alpha_{i}\right\}$. By the (cardinal) ultrapower of the cardinal $\alpha$ modulo $D$, we mean the cardinal $\alpha^{I} / D=\prod_{i \in I} \alpha / D . \quad \beta$ is said to be a (cardinal) $\xi$-limit ultrapower of $\alpha$ if there exist functions $\gamma$, $J, E$ with domain $\xi+1$ such that the following hold:

(i ) $\alpha=\gamma_{0}$;

(ii) $\beta=\gamma_{\xi}$;

(iii) for each $\zeta \leqq \xi, \gamma_{\zeta}$ is a cardinal, $J_{\zeta}$ is a set, and $E_{\zeta}$ is an ultrafilter on $J_{\zeta}$;

(iv) for each $\zeta<\xi, \gamma_{\zeta+1}=\gamma_{\zeta}^{J} \zeta \mid E_{\zeta}$; and

(v) whenever $0<\zeta \leqq \xi, \gamma_{\zeta}=\bigcup_{\eta<\zeta} \gamma_{\eta+1}$.

By the ultraproduct of pairs of cardinals $\left(\alpha_{i}, \beta_{i}\right)$ with $i \in I$, in symbols, $\prod_{i \in I}\left(\alpha_{i}, \beta_{i}\right) / D$, we mean the pair of cardinals $\left(\prod_{i \in I} \alpha_{i} / D, \Pi_{i \in I} \beta_{i} \mid D\right)$. Similarly $(\alpha, \beta)^{I} / D=\left(\alpha^{I} / D, \beta^{I} / D\right)$. The pair of cardinals $\left(\beta, \beta^{\prime}\right)$ is said to be a $\xi$-limit ultrapower of the pair of cardinals $\left(\alpha, \alpha^{\prime}\right)$ if there exist functions $\gamma, \gamma^{\prime}, J, E$, with domain $\xi+1$ such that $\alpha, \gamma, J, E, \beta$ satisfy conditions (i) - (v) and $\alpha^{\prime}, \gamma^{\prime}, J, E, \beta^{\prime}$ satisfy conditions (i) (v). Finally, $S_{\omega}(I)$ denotes the set of all nonempty finite subsets of $I$, i.e., $S_{\omega}(I)=\{s \subseteq I \mid 0<\kappa(s)<\omega\}$.

To conclude this section, we shall prove a preliminary result concerning the products of cardinals.

Lemma 1.1. Let $I \neq 0$ and let $\alpha_{i}$ be infinite cardinals with $i \in I$. For each $i \in I$, let $J_{i}=\left\{j \in I \mid \alpha_{j} \leqq \alpha_{i}\right\}$. Then $\prod_{s \in S_{\omega}(I)} \prod_{i \in s} \alpha_{i}=\prod_{i \in I} \alpha_{i}^{\kappa\left(J_{i}\right)}$.

Proof. Let $A=\left\{\alpha_{i} \mid i \in I\right\}$ and, for each $\alpha \in A$, let $H_{\alpha}=\left\{i \in I \mid \alpha_{i}=\alpha\right\}$ and $S_{\alpha}=\left\{s \in S_{\omega}(I) \mid \prod_{i \in s} \alpha_{i}=\alpha\right\}$. It is easily seen that 


$$
\prod_{s \in S_{\omega}(I)} \prod_{i \in s} \alpha_{i}=\prod_{\alpha \in A} \alpha^{\kappa\left(S_{\alpha a}\right)}
$$

On the other hand

$$
\prod_{i \in I} \alpha_{i}^{\kappa\left(J_{i}\right)}=\prod_{\alpha \in A} \prod_{i \in H_{\alpha}} \alpha^{\kappa\left(J_{i}\right)} .
$$

Let $\alpha \in A$ and $j \in H_{\alpha}$. It is clear that $H_{\alpha} \subseteq J_{i}$ and $J_{j}=J_{i}$ for each $i \in H_{\alpha}$. Thus

$$
\prod_{i \in H_{\alpha}} \alpha^{\kappa\left(J_{i}\right)}=\left(\alpha^{\kappa\left(J_{j}\right)}\right)^{\kappa\left(H_{\alpha}\right)}=\alpha^{\kappa\left(J_{j}\right)} .
$$

To prove the lemma, it is sufficient to show that

$$
\alpha^{\kappa\left(S_{\alpha}\right)}=\alpha^{\kappa\left(J_{j}\right)} .
$$

It is obvious that $\kappa\left(J_{j}\right) \leqq \kappa\left(S_{\alpha}\right)$, and hence

$$
\alpha^{\kappa\left(J_{j}\right)} \leqq \alpha^{\kappa\left(S_{\alpha}\right)} .
$$

Since $\alpha$ is infinite and $S_{\alpha} \subseteq S_{\omega}\left(J_{j}\right)$, we have

$$
\alpha^{\kappa\left(S_{\alpha}\right)} \leqq \alpha^{\kappa\left(S_{\omega}\left(J_{j}\right)\right)} \leqq \alpha^{\kappa\left(J_{j}\right)} .
$$

The lemma is proved.

2. Cardinalities of ultraproducts. We first state a lemma which is an easy consequence of [2, Th. 1.17], and whose proof shall be omitted here.

LeMma 2.1. Let $I \neq 0$ and let $\alpha_{i}$ be cardinals with $i \in I$. Then there exists an ultrafilter $D$ on $S_{\omega}(I)$ such that

$$
\prod_{i \in I} \alpha_{i} \leqq \prod_{s \in S_{\omega}(I)}\left(\prod_{\imath \in s} \alpha_{i}\right) / D
$$

LEMMA 2.2. Let $I \neq 0$ and let $\alpha_{i}$ be infinite cardinals with $i \in I$. Then there exists an ultrafilter $D$ on $S_{\omega} S_{\omega}(I)$ such that

$$
\prod_{s \in S_{\omega}(I)}\left(\prod_{i \in s} \alpha_{i}\right)=\prod_{t \in S_{\omega} S_{\omega}(I)}\left(\prod_{s \in t} \prod_{i \in s} \alpha_{i}\right) / D
$$

Proof. By Lemma 2.1 there exists an ultrafilter $D$ on $S_{\omega} S_{\omega}(I)$ such that

$$
\prod_{s \in S_{\omega}(I)}\left(\prod_{i \in s} \alpha_{i}\right) \leqq \prod_{t \in S_{\omega} S_{\omega}(I)}\left(\prod_{s \in t} \prod_{i \in s} \alpha_{i}\right) / D
$$

It is clear that

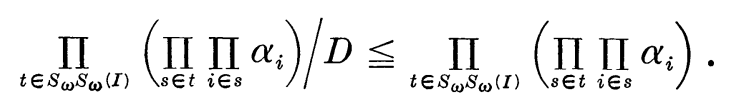


Since each $\alpha_{i}$ is infinite and $t$ is finite, we have

$$
\prod_{t \in S_{\omega} S_{\omega}(I)}\left(\prod_{s \in t} \prod_{i \in s} \alpha_{i}\right)=\prod_{t \in S_{\omega} S_{\omega}(I)}\left(\prod_{i \in \cup t} \alpha_{i}\right) .
$$

Let $J_{s}=\left\{t \in S_{\omega} S_{\omega}(I) \mid \bigcup t=s\right\}$. By the general associative law for cardinal products,

$$
\prod_{t \in S_{\omega} S_{\omega}(I)}\left(\prod_{i \in \cup t} \alpha_{i}\right)=\prod_{s \in S_{\omega}(I)} \prod_{t \in J_{s}} \prod_{i \in s} \alpha_{i} .
$$

Since each $s$ is finite, $J_{s}$ is finite. Again, using the fact that each $\alpha_{i}$ is infinite, we have

$$
\prod_{s \in S_{\omega}(I)} \prod_{t \in J_{s}} \prod_{i \in s} \alpha_{i}=\prod_{s \in S \omega(I)} \prod_{i \in s} \alpha_{i}
$$

Putting (1)-(5) together we obtain the conclusion of the lemma.

Lemma 2.3. Let $\alpha$ be infinite and let $\beta>0$. Then there exists an ultrafilter $D$ on $\beta$ such that $\alpha^{\beta}=\alpha^{\beta} / D$.

Proof. If $\beta$ is finite, then cleary $\alpha^{\beta}=\alpha^{\beta} / D$ for every ultrafilter $D$ on $\beta$. Suppose $\beta$ is infinite. By Lemma 2.1 there exists an ultrafilter $E$ on $S_{\omega}(\beta)$ such that

$$
\alpha^{\beta} \leqq \prod_{s \in S_{\omega}(\beta)}\left(\alpha^{\kappa(s)}\right) / E
$$

Since each $s$ is finite, $\alpha^{\kappa(s)}=\alpha$, and hence

$$
\alpha^{\beta} \leqq \alpha^{S_{\omega}(\beta)} / E \text {. }
$$

Since $\beta$ is infinite, there is a one-to-one correspondence from $S_{\omega}(\beta)$ onto $\beta$, from which we can obtain an ultrafilter $D$ on $\beta$ such that $\alpha^{\beta} \leqq \alpha^{\beta} / D$. It is clear that $\alpha^{\beta} / D \leqq \alpha^{\beta}$, and therefore the conclusion of the lemma holds.

Lemma 2.4. Let $\alpha$ be infinite. Then $\alpha^{\omega \xi}$ is a $\xi$-limit ultrapower of $\alpha$.

Proof. Let $\gamma_{0}=\alpha$. For each $\zeta \leqq \xi$, let $\gamma_{\zeta}=\alpha \omega \zeta, J_{\zeta}=\omega_{\zeta}$, and $E_{\zeta}$ be an ultrafilter on $J_{\zeta}$ such that $\gamma_{\zeta}^{J} \zeta / E_{\zeta}=\gamma_{\zeta}^{\omega \zeta}=\alpha^{\omega}{ }^{\omega}$. Evidently, for $\zeta<\xi, \gamma_{\zeta+1}=\alpha^{\omega} \zeta^{+1}=\alpha^{\omega \zeta}$ and hence $\gamma_{\zeta+1}=\gamma_{\zeta}^{J \zeta} / E_{\zeta}$. Suppose that $0<\zeta \leqq \xi$. Then $\gamma_{\zeta}=\alpha^{\omega \zeta}=\sum_{\eta<\zeta} \alpha^{\omega_{\eta}}=\bigcup_{\eta<\zeta} \alpha^{\omega_{\eta}}=\bigcup_{\eta<\zeta} \gamma_{\eta+1}$. The proof is complete.

3. Classes of pairs of cardinals. Let $M$ be a class of pairs of infinite cardinals. 
Theorem 3.1. Suppose $M$ is closed under ultraproducts. If $I \neq 0$ and $\left\{\left(\prod_{i \in s} \alpha_{i}, \prod_{i \in s} \beta_{i}\right) \mid s \in S_{\omega}(I)\right\} \subseteq M$, then $\left(\prod_{s \in S_{\omega}(I)} \prod_{i \in s} \alpha_{i}, \prod_{s \in S_{\omega}(I)} \Pi_{i \in s} \beta_{i}\right) \in M$.

Proof. We first show that

$$
\text { for each } t \in S_{\omega} S_{\omega}(I),\left(\prod_{s \in t} \prod_{\imath \in s} \alpha_{i}, \prod_{s \in t} \prod_{i \in s} \beta_{i}\right) \in M \text {. }
$$

Since $\alpha_{i}, \beta_{i}$ are all infinite and $t, s$ are finite, we have

$$
\prod_{s \in t} \prod_{i \in s} \alpha_{i}=\prod_{i \in \cup t} \alpha_{i}
$$

and

$$
\prod_{s \in t} \prod_{i \in s} \beta_{i}=\prod_{i \in \cup t} \beta_{i} .
$$

Since $\mathrm{U} t \in S_{\omega}(I)$, (1) follows. Now, the conclusion of the theorem follows from (1) and Lemma 2.2.

THEOREM 3.2. Suppose $M$ is closed under ultraproducts. Suppose further that $I \neq 0,\left\{\left(\alpha_{i}, \beta_{i}\right) \mid i \in I\right\} \subseteq M$, and for every $i, j \in I, \alpha_{i}<\alpha_{j}$ implies $\beta_{i} \leqq \beta_{j}$. Then

$$
\left(\prod_{s \in S_{\omega}(I)} \prod_{i \in s} \alpha_{i}, \prod_{s \in S_{\omega}(I)} \prod_{i \in s} \beta_{i}\right) \in M .
$$

Proof. By Theorem 3.1 it is sufficient to prove that

$$
\left(\prod_{i \in s} \alpha_{i}, \prod_{i \in s} \beta_{i}\right) \in M \text { for each } s \in S_{\omega}(I) .
$$

Let $s \in S_{\omega}(I)$. Let $s_{1}=\left\{j \in s \mid \prod_{i \in s} \alpha_{i}=\alpha_{j}\right\}$ and $s_{2}=\left\{j \in s \mid \prod_{i \in s} \beta_{i}=\beta_{j}\right\}$. Since $s$ is finite, $s_{1} \neq 0$ and $s_{2} \neq 0$. We show that $s_{1} \cap s_{2} \neq 0$. Assume $s_{1} \cap s_{2}=0$. Let $j \in s_{1}$ and $k \in s_{2}$. Since $\alpha_{k}<\alpha_{j}$, we have $\beta_{k} \leqq \beta_{j}$. On the other hand, $\beta_{j}<\beta_{k}$, and this is a contradiction. Let $j \in s_{1} \cap s_{2}$; then

$$
\left(\prod_{i \in s} \alpha_{i}, \prod_{i \in s} \beta_{i}\right)=\left(\alpha_{j}, \beta_{j}\right) \in M,
$$

and (1) holds. The theorem is proved.

COROLLARY 3.3. Suppose $M$ is closed under ultraproducts. Let $\alpha_{\zeta}, \beta_{\zeta}, \zeta<\xi$, be such that $\left\{\left(\alpha_{\zeta}, \beta_{\zeta}\right) \mid \zeta<\xi\right\} \subseteq M$, and whenever $\eta<\zeta<\xi$, $\alpha_{\eta}<\alpha_{\zeta}$ and $\beta_{\eta}<\beta_{\zeta}$. Then

$$
\left(\prod_{\zeta<\xi} \alpha_{\zeta}^{\kappa(\zeta)}, \prod_{\zeta<\xi} \beta_{\zeta}^{\kappa(\zeta)}\right) \in M
$$


Proof. By Lemma 1.1 and Theorem 3.2.

COROLlaRY 3.4. Suppose $M$ is closed under ultraproducts. Let $\alpha_{0}, \alpha_{1}, \alpha_{2}, \cdots, \beta_{0}, \beta_{1}, \beta_{2}, \cdots$, be strictly increasing sequences such that $\left\{\left(\alpha_{n}, \beta_{n}\right) \mid n \in \omega\right\} \subseteq M$. Then $\left(\Pi_{n<\omega} \alpha_{n}, \Pi_{n<\omega} \beta_{n}\right) \in M$.

Proof. By Corollary 3.3.

THEOREM 3.5. Suppose $M$ is closed under ultrapowers. If $(\alpha, \beta) \in M$, then $\left(\alpha^{\gamma}, \beta^{\gamma}\right) \in M$.

Proof. By Lemma 2.3.

THEOREM 3.6. Suppose $M$ is closed under $\xi$-limit ultrapowers. If $(\alpha, \beta) \in M$ then $\left(\alpha^{\omega \xi \xi}, \beta^{\omega \xi}\right) \in M$.

Proof. By Lemma 2.4 and its proof.

The results obtained so far in this section in Corollary 3.3 and Theorems 3.5 and 3.6 can be stated more simply if we assume the Generalized Continuum Hypothesis. The reason for this is because the operations of cardinal powers and cardinal products become more transparent. For the remainder of this section, we assume the Generalized Continuum Hypothesis.

THEOREM 3.7. Suppose $M$ is closed under ultrapowers. Let $(\alpha, \beta) \in M$. Then the following hold:

(i ) If $c f(\alpha)=c f(\beta)$, then $\left(\alpha^{+}, \beta^{+}\right) \in M$.

(ii) If $c f(\alpha)>c f(\beta)$, then $\left(\alpha, \beta^{+}\right) \in M$.

(iii) If $c f(\alpha)<c f(\beta)$, then $\left(\alpha^{+}, \beta\right) \in M$.

Proof. It is known that $\alpha<\alpha^{\gamma}$ if and only if $c f(\alpha) \leqq \gamma$. By the Generalized Continuum Hypothesis, we see that $\alpha^{c f(\alpha)}=\alpha^{+}$. Hence the conclusions of the theorem follow from Theorem 3.5.

Examples. Suppose $M$ is closed under ultrapowers. If $\left(\omega_{\omega}, \omega\right) \in M$ then $\left(\omega_{\omega+1}, \omega_{1}\right) \in M$. If $\left(\omega_{2}, \omega\right) \in M$ then $\left(\omega_{2}, \omega_{1}\right) \in M$. If $\left(\omega_{\omega}, \omega_{1}\right) \in M$ then $\left(\omega_{\omega+1}, \omega_{1}\right) \in M$.

THEOREM 3.8. Suppose $M$ is closed under $\xi$-limit ultrapowers. Let $(\alpha, \beta) \in M$. Then the following hold:

(i) If $\alpha<\gamma$ and $\beta<\gamma$, then $(\gamma, \gamma) \in M$. 
(ii) If $\beta<\gamma \leqq c f(\alpha)$, then $(\alpha, \gamma) \in M$.

(iii) If $\alpha<\gamma \leqq c f(\beta)$, then $(\gamma, \beta) \in M$.

Proof. By the Generalized Continuum Hypothesis, if $\gamma \leqq c f(\alpha)$ then $\alpha^{\gamma}=\alpha$, and if $\gamma>\alpha$, then $\alpha^{\gamma}=\gamma$. The conclusions follow from Theorem 3.6.

ExAMPLES. Suppose $M$ is closed under $\xi$-limit ultrapowers. If $\left(\omega_{1}, \omega\right) \in M$ then $\left(\omega_{\omega}, \omega_{\omega}\right) \in M$. If $\left(\omega_{\omega+1}, \omega_{1}\right) \in M$ then $\left(\omega_{\omega+1}, \omega_{\omega}\right) \in M$.

THEOREM 3.9. Suppose $M$ is closed under ultraproducts. Then the following hold:

(i) If $c f(\alpha)=c f(\beta)$ and for every $\gamma<\alpha, \delta<\beta$, there exist $\gamma^{\prime}, \delta^{\prime}$ such that $\gamma<\gamma^{\prime}<\alpha, \delta<\delta^{\prime}<\beta$, and $\left(\gamma^{\prime}, \delta^{\prime}\right) \in M$, then $\left(\alpha^{+}, \beta^{+}\right) \in M$.

(ii) If $\operatorname{cf}(\alpha)<\beta$ and for every $\gamma<\alpha$, theere exists $\gamma^{\prime}$ such that $\gamma<\gamma^{\prime}<\alpha$ and $\left(\gamma^{\prime}, \beta\right) \in M$, then $\left(\alpha^{+}, \beta\right) \in M$.

(iii) If $\operatorname{cf}(\beta)<\alpha$ and for every $\delta<\beta$ there exists $\delta^{\prime}$ such that $\delta<\delta^{\prime}<\beta$ and $\left(\alpha, \delta^{\prime}\right) \in M$, then $\left(\alpha, \beta^{+}\right) \in M$.

Proof. By the Generalized Continuum Hypothesis, the cardinal product of any $c f(\alpha)$-termed sequence of cardinals whose union is $\alpha$ is $\alpha^{+}$. The conclusions follow from applications of this remark and Corollary 3.3.

ExAmPles. Suppose $M$ is closed under ultraproducts. If $\left\{\left(\omega_{\omega+n}, \omega_{n}\right) \mid n \in \omega\right\} \subseteq M$, then $\left(\omega_{\omega+\omega+1}, \omega_{\omega+1}\right) \in M$. If $\left\{\left(\omega_{n+1}, \omega_{1}\right) \mid n \in \omega\right\} \subseteq M$, then $\left(\omega_{\omega+1}, \omega_{1}\right) \in M$.

4. Applications to model theory. We shall now give a brief introduction to those portions of the theory of models which are pertinent to this section.

By a similarity type, or briefly a type, we mean a function $T$ whose domain is a cardinal different from 0 and whose range is included in $\omega$. Let $T$ be a type such that $T(0)=1$ and let $\delta$ be the domain for $T$. A system $\mathfrak{A}=\left\langle A, R_{\xi}\right\rangle_{\xi<\delta}$ is said to be a structure of type $T$ if $A \neq 0$, and, for each $\xi<\delta, R_{\xi}$ is a $T(\xi)$-ary relation over $A$.

Let $L(T)$ be the first-order predicate logic with identity symbol =, an infinite sequence of individual variables $v_{0}, v_{1}, v_{2}, \cdots$, a $T(\xi)$-placed predicate symbol $P_{\xi}$ for each $\xi<\delta$, the usual symbols for propositional connectives and quantifiers, and no predicate or functional variables or individual constants. We assume the definitions of formula and sentence are known, as well as the notion of a sentence of $L(T)$ holding in a structure of type $T$. A class $\boldsymbol{K}$ of structures of type $T$ is said to be 
an elementary class if there exists a set $\Gamma$ of sentences of $L(T)$ such that a structure $\mathfrak{A}$ belongs to $\boldsymbol{K}$ if and only if every sentence of $\Gamma$ holds in $\mathfrak{A}$. A class $\boldsymbol{K}$ of structures of type $T$ is said to be elementarily closed if whenever $\mathfrak{A} \in \boldsymbol{K}$ and every sentence of $L(T)$ holding in $\mathfrak{A}$ holds in $\mathfrak{B}$, then $\mathfrak{B} \in \boldsymbol{K}$ (or, equivalently, $\boldsymbol{K}$ is a union of elementary classes). Notice that every elementary class is elementarily closed.

By the pair of cardinals for a structure $\left\langle A, R_{\xi}\right\rangle_{\xi<\delta}$ we mean the pair $\left(\kappa(A), \kappa\left(R_{0}\right)\right)$. We let $M(\boldsymbol{K})=\{(\alpha, \beta) \mid \alpha, \beta$ are infinite and there exists $\mathfrak{A} \in \boldsymbol{K}$ such that $(\alpha, \beta)$ is the pair of cardinals for $\mathfrak{A}\}$. Notice that if $(\alpha, \beta) \in M(\boldsymbol{K})$, then $\alpha \geqq \beta$.

The following lemmas are easy consequences of known results in the literature (see [2] and [4]).

LEMMA 4.1. If $\boldsymbol{K}$ is an elementary class, then $M(\boldsymbol{K})$ is closed under ultraproducts, ultrapowers, and $\xi$-limit ultrapowers.

Lemma 4.2. If $\boldsymbol{K}$ is elementarily closed, then $M(\boldsymbol{K})$ is closed under ultrapowers and $\xi$-limit ultrapowers.

In view of these two lemmas, we have the following model-theoretic applications of the results of $\S 3$ :

(A) If $\boldsymbol{K}$ is an elementary class, then all results $3.1-3.9$ of $\S 3$ apply to $M(\boldsymbol{K})$.

(B) If $\boldsymbol{K}$ is an elementarily closed class, then the results 3.5-3.8 of $\S 3$ apply to $M(\boldsymbol{K})$.

Lemmas 4.1 and 4.2, and thus the statements (A) and (B), can be somewhat improved. This is done by substituting the notion of an elementary class by the more general notion of a pseudo-elementary class (i.e., $\mathbf{P C}_{4}$, see [2] and [7]) in Lemma 4.1, and substituting the elementarily closed class by the more general union of pseudo-elementary classes in Lemma 4.2. Moreover, for any structure $\mathfrak{A}$, the class $\boldsymbol{K}$ of all structures which are isomorphic to elementary extensions of $\mathfrak{A}$ has the property that $M(\boldsymbol{K})$ is closed under both ultraproducts and $\xi$-limit ultrapowers. Therefore both (A) and (B) are valid for such classes $\boldsymbol{K}$.

We shall now state some earlier theorems formulated in terms of pairs of cardinals which will give some idea of how our results (A) and (B) stand with respect to what was previously known concerning $M(\boldsymbol{K})$. These earlier results differ from ours in that they depend on $\delta$, the domain of the similarity type $T$.

(C) (Löwenheim-Skolem-Tarski) Let $\boldsymbol{K}$ be an elementarily closed class. Let $(\alpha, \beta) \in M(\boldsymbol{K})$ and let $\gamma$ be an infinite cardinal such that $\delta \leqq \gamma$. Then $(\gamma, \gamma) \in M(\boldsymbol{K})$. Furthermore, if $\beta \leqq \gamma \leqq \alpha$, then $(\gamma, \beta) \in M(\boldsymbol{K})$.

(D) (Vaught [8]) Let $\boldsymbol{K}$ be an elementarily closed class. Let $\delta \leqq \omega$ 
and let $\alpha \neq \beta$. If $\left(\alpha, \beta \in M(\boldsymbol{K})\right.$ then $\left(\omega_{1}, \omega_{0}\right) \in M(\boldsymbol{K})$.

The following is a corollary of (A) and (C).

(E) Assume the Generalized Continuum Hypothesis. Let $\boldsymbol{K}$ be an elementary class. If $\delta \leqq \alpha$, cf $(\alpha)<\beta$, and for every $\gamma<\alpha$ there exists $\gamma^{\prime}$ such that $\gamma<\gamma^{\prime}<\alpha$ and $\left(\gamma^{\prime}, \beta\right) \in M(\boldsymbol{K})$, then $(\alpha, \beta) \in M(\boldsymbol{K})$.

Results similar to (E), but depending on (A) alone, follow from Theorems 3.7-3.9.

The following is a corollary of (B) and (C).

(F) Assume the Gereralized Continuum Hypothesis. Let $\boldsymbol{K}$ be an elementarily closed class, and let $(\alpha, \beta) \in M(\boldsymbol{K})$. If $\beta \leqq \gamma^{\prime} \leqq \gamma \leqq \alpha$ and $\delta \leqq \gamma$, then $\left(\gamma, \gamma^{\prime}\right) \in M(\boldsymbol{K})$.

Proof. If $\beta=\gamma^{\prime}$, then $\left(\gamma, \gamma^{\prime}\right) \in M(\boldsymbol{K})$ by (C). On the other hand, if $\beta<\gamma^{\prime}$, then it follows from the Generalized Continuum Hypothesis that $\beta^{\gamma^{\prime}}=\gamma^{\prime}$. Therefore by Theorem 3.6 and $(\mathrm{B}),\left(\alpha^{\gamma^{\prime}}, \gamma^{\prime}\right) \in M(\boldsymbol{K})$. Since $\gamma^{\prime} \leqq \gamma \leqq \alpha^{\gamma^{\prime}}$, we conclude from (C) that $\left(\gamma, \gamma^{\prime}\right) \in M(\boldsymbol{K})$.

5. Some negative results and open problems. In $\S 4$ we presented some positive results on $M(\boldsymbol{K})$ when $\boldsymbol{K}$ is an elementary class. In this section we shall give some negative results stating that certain other plausible conjectures about $M(\boldsymbol{K})$ where $\boldsymbol{K}$ is elementary have counterexamples. We conclude this section by stating some natural open problems.

One can easily construct an example of an elementary class $\boldsymbol{K}$ such that $(\alpha, \beta) \in M(\boldsymbol{K})$ if and only if $\omega \leqq \beta=\alpha$.

We shall now give an example of an elementary class $\boldsymbol{K}$ such that $(\alpha, \beta) \in M(K)$ if and only if $\omega \leqq \beta \leqq \alpha \leqq 2^{\beta}$. (This example is due to R. M. Robinson and was privately communicated to R. L. Vaught, from whom the authors learned of it.)

Let $\delta=2, T(0)=1$, and $T(1)=2$. Let $K$ be the elementary class characterized by the sentence

$$
\forall x, y\left[x \neq y \rightarrow \exists z\left\{P_{0}(z) \wedge \neg\left(P_{1}(x, z) \longleftrightarrow P_{1}(y, z)\right)\right\}\right] .
$$

Thus, if $\left\langle A, R_{0}, R_{1}\right\rangle \in \boldsymbol{K}$, the mapping $f(a)=\left\{b \mid R_{0}(b)\right.$ and $\left.R_{1}(a, b)\right\}$ is a one-to-one mapping of $A$ into the set of all subsets of $R_{0}$. From this we see that $M(\boldsymbol{K})$ is the desired class of pairs of cardinals.

From this example we easily see that the following hold. Let $\beta_{0}=\beta$ and, for each $n<\omega$, let $\beta_{n+1}=2^{\beta_{n}}$. Then for each $n$ there exists an elementary class $\boldsymbol{K}$ such that $(\alpha, \beta) \in M(\boldsymbol{K})$ if and only if $\omega \leqq \beta \leqq \alpha \leqq \beta_{n}$.

We may also easily give an example, when $\omega \leqq \beta \leqq \alpha$, of a type $T$ (with $\delta=\alpha$ ) and elementary class $K$ such that $\left(\alpha^{\prime}, \beta^{\prime}\right) \in M(K)$ if and 
only if $\alpha \leqq \alpha^{\prime}, \beta \leqq \beta^{\prime}$, and $\beta^{\prime} \leqq \alpha^{\prime}$.

For the last collection of examples we need the following restatement of a lemma in [4].

A cardinal $\beta$ is said to be nonmeasurable if every countably complete ultrafilter on $\beta$ is principal. A structure $\mathfrak{A}=\left\langle A, R_{\xi}\right\rangle_{\xi<\delta}$ of type $T$ is said to be complete (see [5]) if, for every finitary relation $R$ on $A$, we have $R \in\left\{R_{\xi} \mid \xi<\delta\right\}$. Notice that if $\mathfrak{A}$ if complete, then $\delta \geqq 2^{\kappa(A)}$.

Lemma 5.1. Suppose $\mathfrak{A}$ is complete, $\boldsymbol{K}$ is the smallest elementary class containing $\mathfrak{A}, \alpha=\kappa(A) \omega \leqq \beta=\kappa\left(R_{0}\right)$. Then the following hold:

( i ) Suppose $\alpha$ is nonmeasurable and $\left(\alpha^{\prime}, \beta^{\prime}\right) \in M(K)$. Then either $\alpha^{\prime}=\alpha$ and $\beta^{\prime}=\beta$, or $\alpha^{\prime} \geqq \alpha^{\omega}$ and $\beta^{\prime} \geqq \beta^{\omega}$.

(ii) Suppose $\beta$ is nonmeasurable and $\left(\alpha^{\prime}, \beta^{\prime}\right) \in M(K)$. Then either $\beta^{\prime}=\beta$, or $\alpha^{\prime} \geqq \alpha^{\omega}$ and $\beta^{\prime} \geqq \beta^{\omega}$.

TheOREM 5.2. Suppose $\omega \leqq \beta \leqq \alpha$. Then the following hold:

(i) Suppose $\alpha$ is nonmeasurable, and $\beta<\beta^{\omega}$. Then there exist a type $T$ and an elementary class $\boldsymbol{K}$ such that $\left(\alpha^{\prime}, \beta\right) \in M(K)$ if and only if $\alpha^{\prime}=\alpha$.

(ii) Suppose $\beta$ is nonmeasurable and $\alpha<\alpha^{\omega}$. Then there exists a type $T$ and an elementary class $\boldsymbol{K}$ such that $\left(\alpha, \beta^{\prime}\right) \in M(\boldsymbol{K})$ if and only if $\beta^{\prime}=\beta$.

(iii) Suppose $\alpha$ is nonmeasurable, $\alpha<\alpha^{\omega}$, and $\beta<\beta^{\omega}$. Then there exist a type $T$ and an elementary class $\boldsymbol{K}$ such that $\left(\alpha, \beta^{\prime}\right) \in M(\boldsymbol{K})$ if and only if $\beta^{\prime}=\beta$, and $\left(\alpha^{\prime}, \beta\right) \in M(K)$ if and only if $\alpha^{\prime}=\alpha$.

\section{Proof. By Lemma 5.1.}

Open problems. Let $\boldsymbol{K}$ be an arbitrary elementary class and $\delta \leqq \omega$. I. Does $\left(\omega_{\xi+\omega+\zeta}, \omega_{\xi}\right) \in M(K)$ and $\omega \leqq \beta \leqq \alpha$ imply $(\alpha, \beta) \in M(K)$ ?

II. Does $\left(\omega_{\xi+\eta}, \omega_{\xi}\right) \in M(K)$ imply $\left(\omega_{\zeta+\eta}, \omega_{\zeta}\right) \in M(K)$ ?

III. Does $(\alpha, \beta) \in M(K)$ imply $\left(2^{\alpha}, 2^{\beta}\right) \in M(K)$ ?

IV. Does $\left\{\left(\omega_{n}, \omega_{0}\right) \mid n<\omega\right\} \subseteq M(\boldsymbol{K})$ imply $\left(\omega_{\omega}, \omega_{0}\right) \in M(\boldsymbol{K})$ ?

I'. Does $\left(\omega_{\omega}, \omega_{0}\right) \in M(K)$ imply $\left(\omega_{\omega+1}, \omega_{0}\right) \in M(K)$ ?

Does $\left(\omega_{\omega+1}, \omega_{1}\right) \in M(\boldsymbol{K})$ imply $\left(\omega_{\omega+2}, \omega_{1}\right) \in M(K)$ ?

II'. Does $\left(\omega_{1}, \omega_{0}\right) \in M(K)$ imply $\left(\omega_{2}, \omega_{1}\right) \in M(K)$ ?

Does $\left(\omega_{3}, \omega_{2}\right) \in M(K)$ imply $\left(\omega_{2}, \omega_{1}\right) \in M(K)$ ?

\section{REFERENCES}

1. C. C. Chang and H. J. Keisler, Pairs of cardinals for models of a given theory, Abstract 61T-134, Notices of AMS, 8 (1961), 275.

2. T. Frayne, Anne C. Morel and D. Scott, Reduced direct products, to appear in Fund. Math.

3. H. J. Keisler, Ultraproducts and elementary classes, Indag. Math., 23 (1961), 477-495. 
4. H. J. Keisler, Limit ultrapowers, submitted for publication in the Trans. of AMS.

5. M Rabin, Arithmetical extensions with prescribed cardinality, Indag. Math., 21 (1959), 439-446.

6. A. Tarski, Quelques théorèmes sur les alephs, Fund. Math., 7 (1925), 1-14.

7. A. Tarski, Contributions to the theory of models, I and II, Indag. Math, 16 (1954), 572-588.

8. R. L. Vaught, A Löwenheim-Skolem theorem for two cardinals, Abstract 578-58, Notices of AMS, 8 (1961), 239.

UNIVERSity OF CALIFornia, LOS ANGELES

UNIVERSITY OF CALIFORNIA, BERKELEY, AND

Institute FOR Defense ANAlyses, PRINCEton. 



\title{
PACIFIC JOURNAL OF MATHEMATICS
}

\author{
EDITORS
}

RalPh S. Phillips

Stanford University

Stanford, California

M. G. Arsove

University of Washington

Seattle 5 , Washington
A. L. Whiteman

University of Southern California Los Angeles 7, California

LOWell J. PAIGE

Unıversity of California

Los Angeles 24, California

\section{ASSOCIATE EDITORS}
E. F. BECKENBACH
D. DERRY
H. L. ROYDEN
E. G. STRAUS
T. M. CHERRY
M. OHTSUKA
E. SPANIER
F. WOLF

\section{SUPPORTING INSTITUTIONS}

\author{
UNIVERSITY OF BRITISH COLUMBIA \\ CALIFORNIA INSTITUTE OF TECHNOLOGY \\ UNIVERSITY OF CALIFORNIA \\ MONTANA STATE UNIVERSITY \\ UNIVERSITY OF NEVADA \\ NEW MEXICO STATE UNIVERSITY \\ OREGON STATE UNIVERSITY \\ UNIVERSITY OF OREGON \\ OSAKA UNIVERSITY \\ UNIVERSITY OF SOUTHERN CALIFORNIA
}

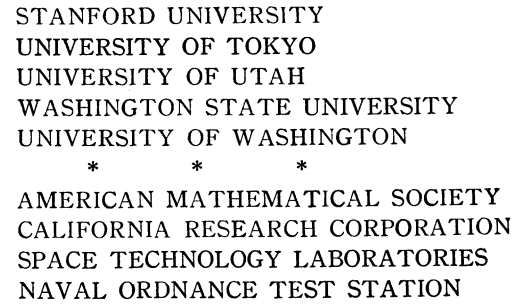

Mathematical papers intended for publication in the Pacific Journal of Mathematıcs should be typewritten (double spaced), and the author should keep a complete copy. Manuscripts may be sent to any one of the four editors. All other communications to the editors should be addressed to the managing editor, L. J. Paige at the University of California, Los Angeles 24, California.

50 reprints per author of each article are furnıshed free of charge; additional copies may be obtained at cost in multiples of 50 .

The Pacific Journal of Mathematics is published quarterly, in March, June, September, and December. Effective with Volume 13 the price per volume (4 numbers) is $\$ 18.00$; single issues, $\$ 5.00$. Special price for current issues to individual faculty members of supporting institutions and to individual members of the American Mathematical Society: $\$ 8.00$ per volume; single issues $\$ 2.50$. Back numbers are available.

Subscriptions, orders for back numbers, and changes of address should be sent to Pacific Journal of Mathematics, 103 Highland Boulevard, Berkeley 8, California.

Printed at Kokusai Bunken Insatsusha (International Academic Printing Co., Ltd.), No. 6 , 2-chome, Fujimi-cho, Chiyoda-ku, Tokyo, Japan.

PUBLISHED BY PACIFIC JOURNAL OF MATHEMATICS, A NON-PROFIT CORPORATION

The Supporting Institutions listed above contribute to the cost of publication of this Journal, but they are not owners or publishers and have no responsibility for its content or policies. 


\section{Pacific Journal of Mathematics}

\section{Vol. 12, No. $3 \quad$ March, 1962}

Alfred Aeppli, Some exact sequences in cohomology theory for Kähler

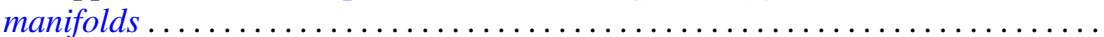

Paul Richard Beesack, On the Green's function of an N-point boundary value

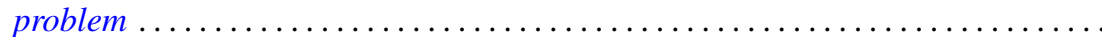

James Robert Boen, On p-automorphic p-groups....

James Robert Boen, Oscar S. Rothaus and John Griggs Thompson, Further results

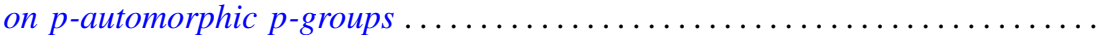

James Henry Bramble and Lawrence Edward Payne, Bounds in the Neumann problem for second order uniformly elliptic operators ..................

Chen Chung Chang and H. Jerome (Howard) Keisler, Applications of ultraproducts of pairs of cardinals to the theory of models .........................

Stephen Urban Chase, On direct sums and products of modules ................

Paul Civin, Annihilators in the second conjugate algebra of a group algebra .......

J. H. Curtiss, Polynomial interpolation in points equidistributed on the unit

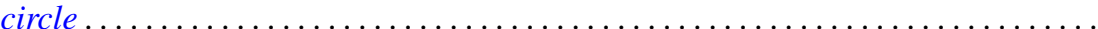

Marion K. Fort, Jr., Homogeneity of infinite products of manifolds with

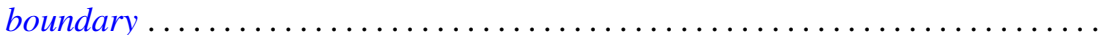

James G. Glimm, Families of induced representations . . . . . . . . . . . . . . .

Daniel E. Gorenstein, Reuben Sandler and William H. Mills, On almost-commuting

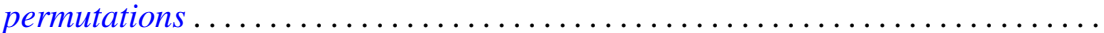

Vincent C. Harris and M. V. Subba Rao, Congruence properties of $\sigma_{r}(N) \ldots \ldots \ldots$

Harry Hochstadt, Fourier series with linearly dependent coefficients . . . . . . . . . . 925

Kenneth Myron Hoffman and John Wermer, A characterization of $C(X)$. .

Robert Weldon Hunt, The behavior of solutions of ordinary, self-adjoint differential equations of arbitrary even order...

Edward Takashi Kobayashi, A remark on the Nijenhuis tensor

David London, On the zeros of the solutions of $w^{\prime \prime}(z)+p(z) w(z)=0$

Gerald R. Mac Lane and Frank Beall Ryan, On the radial limits of Blaschke products...

T. M. MacRobert, Evaluation of an E-function when three of its upper parameters

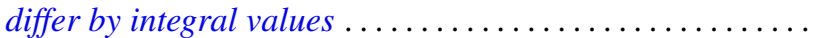

Robert W. McKelvey, The spectra of minimal self-adjoint extensions of a symmetric operator

Adegoke Olubummo, Operators of finite rank in a reflexive Banach space. .

David Alexander Pope, On the approximation of function spaces in the calculus of variations

Bernard W. Roos and Ward C. Sangren, Three spectral theorems for a pair of singular first-order differential equations...............

Arthur Argyle Sagle, Simple Malcev algebras over fields of characteristic zero .

S. C. Tang, Some theorems on the ratio of empirical distribution to the theoretical distribution

Robert Charles Thompson, Normal matrices and the normal basis in abelian number fields.

Howard Gregory Tucker, Absolute continuity of infinitely divisible distributions ... 